\title{
Smart Protective Protection Equipment for an accessible work environment and occupational hazard prevention
}

\author{
MÁRQUEZ SÁNCHEZ, Sergio \\ Grupo de investigación en Bioinformática, Sistemas \\ Informáticos Inteligentes y Tecnología Educativa (BISITE) \\ Edificio Multiusos I+D+I: Calle Espejo s/n, 37007, \\ Salamanca, Spain \\ smarquez@usal.es
}

\author{
CORCHADO RODRIGUEZ, Juan Manuel \\ Grupo de investigación en Bioinformática, Sistemas \\ Informáticos Inteligentes y Tecnología Educativa (BISITE) \\ Edificio Multiusos I+D+I: Calle Espejo s/n, 37007, \\ Salamanca, Spain \\ jm@corchado.net
}

\begin{abstract}
Wearable technologies have begun to play an important role in the workplace. This paper presents a Smart PPE (Personal Protective Equipment) solution which employs a sensor network located on a helmet and a belt to monitor the state of the worker and the environment. Most of the accidents that occur in the workplace are caused by the lack of prevention measures, poor safety training and obsolete safety systems which do no adapt technologically to the needs of today's work environments. The solutions provided by Industry 4.0 for hazard prevention and propose a wireless PPE model that incorporates intelligent tools and fabrics capable of reacting in real time to a risk situation. This novel model implements continuous risk monitoring biometrics of the worker, detects the external impact, shock, luminosity, gases, temperature of the environment and provides real-time recommendations to workers. The motivation behind this work is to improve health and safety in work sectors with high accident risk.
\end{abstract}

Keywords- e-Health , Context Modelling, Condition monitoring

\section{INTRODUCTION}

According to figures collected by the Spanish General Workers Union (GWU) in September last year, every day 2 people have died as a result of an accident at work, 11 have suffered a serious accident, 1391 a minor one and 1,954 an accident that did not require them to go on sick leave. The statistics for the first seven months of 2018 are alarming, with a total of 359 deaths and 773,926 occupational accidents in Spain, there have been 6,147 occupational accidents more than in 2017, representing a $0.8 \%$ increase. Accidents occurring during the workday increased by $3.3 \%$ while the accidents on the way to or from work increased by $4.7 \%$. We examined the high accident rate and traced back its origin, arriving at the conclusion that the long period of crisis has led to budget cuts, lack of safety measures and the hiring of inexperienced workers on temporary contracts; all these significantly contribute to the increase in work accidents. Budget cuts mean that employers cannot afford to provide proper safety training to their workers, which is essential in accident prevention.

Through a careful analysis of the accidents that caused workers to go on sick leave, we can identify overexertion of the musculoskeletal system as a major cause; in more than 15,000 cases $(38 \%)$. Furthermore, the sectors with the highest incidence rate include the industrial sector (manufacturing) with 59,323 accidents followed by trade with 42,296 and construction with 38,543 . More than a third of occupational accidents are directly related to i) the lack of training in safety, health and prevention, ii) the lack of availability, adaptation and/or training in the use of protective equipment and iii) the absence of clearly defined safety procedures for the handling of tools and/or the execution of tasks. Fig.1 below shows a graph with the main causes of accidents.

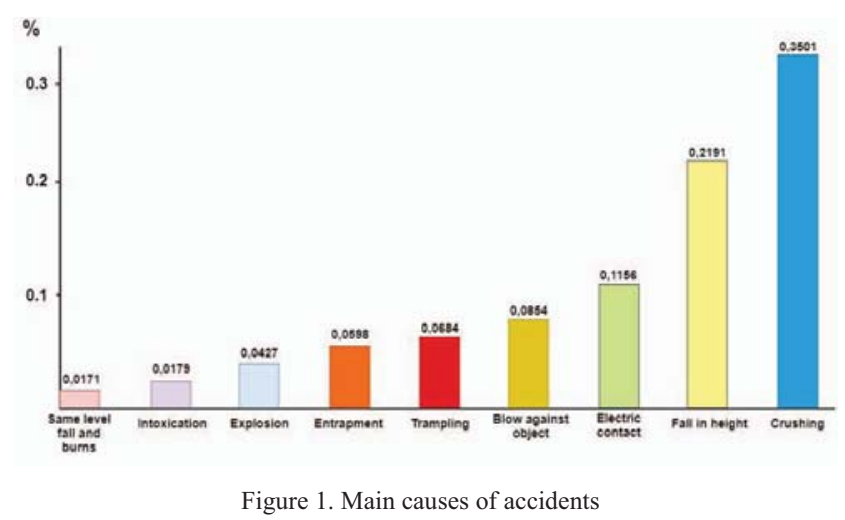

Today's technological advances make it possible to approach these problems effectively, leaving behind the traditional methods of safety training and the obsolete personal protective equipment (PPE), which is disconnected from the real working environment and does not adapt to the individual needs of each worker.

The proposed solution can reduce the costs of training or prevention and how this is a realistic solution in the context of strongly reduced budgets.

Hence, this proposal aims to provide an integral solution to improve safety and health in the workplace, providing an innovative tool in the form of personal protective equipment (PPE) with the capacity to respond in real time to the risks 
present in the workplace, developing a comprehensive, personalized system adapted to both the environment, as wel as to the personal circumstances and capabilities of each worker. A schema of the system is shown in Fig.2 by performing an intelligent measurement of parameters implemented in PPE we can feed a system and by means of Artificial Intelligence (AI) we can favour our strategy of occupational risk prevention. The helmet and belt incorporate a suitable technological framework through the use of intelligent fabrics, microelectronics and advanced sensors.

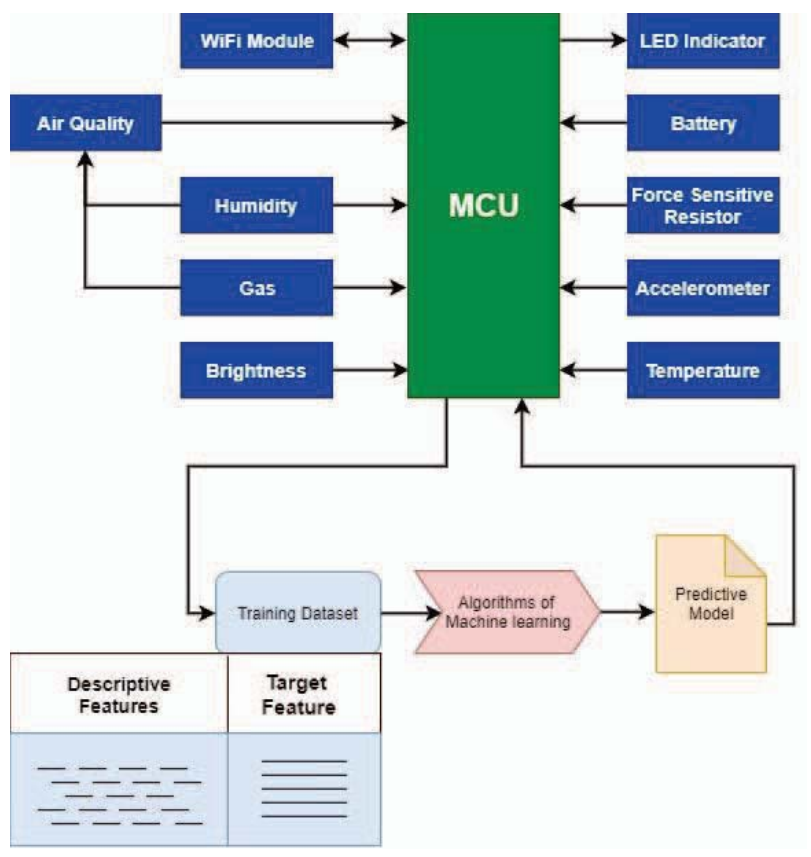

Figure 2. Summary diagram of system opertion

This system proposes solutions that cover the range of actions involved in the occupational risk prevention strategy, providing advanced training to workers to improve their safety (e.g. sensors alert the worker of risk situations) and health at work. In addition, the system makes the working environment accessible, adapting to the physical, cognitive and sensory conditions of each worker, e.g. haptic technology makes it possible for people with visual and hearing impairments to identify the potential risks in their environment.

The rest of the paper is organized as follows: Sect. 2 describes the related work; Sect. 3 presents the proposed method; Sect. 4 give the results and the corresponding analysis, and conclusions arrived and future works are given in Sect. 5.

\section{RELATED WORK}

Creating a safe and healthy working environment is a great concern within the business world, especially in the industrial sector. In this regard, numerous studies have concluded that the incorporation of technology in different industrial sectors can significantly reduce the number of accidents. A wide range of safety and health risks can be encountered depending on the type of work. However, all of them can be covered through the design of adequate personal protection equipment using intelligent textiles. PPE captures different parameters, such as temperature, humidity, electromagnetic field, radiation, movements, forces, biological activity, etc., and is capable of sending signals to the worker on the basis of those values.

The first intelligent PPE has been designed in the 1990s as part of researches on military, aerospace and wearable technology fields [10-15]. The possibility of attaching electronic equipment to the body was the key focus of those researches and thanks to the advances in today's technology, the first commercial products, such as wearable devices and intelligent textiles have begun to appear in the market. The current trend in the design of uniforms for professionals such as firefighters, soldiers and workers is to use technology to adapt the uniforms to their particular needs. Nonetheless, there are also devices, such as smart-watch, that are designed to adapt to several professions.

Several outstanding state-of-the-art researches have been used as a reference for this work. On the one hand, we focus on advances in the area of sensors, which have greatly contributed to the development of sensor applications [2][3][4] On the other hand, we analyse the use of Smart Materials embedded in PPE, such as PCM phase change microcapsules that react to a sudden change in temperature [5] or the possibility of generating electricity through movement [6]. Compatibility, interoperability, modularity, ergonomics, etc. are important factors that must be considered in the design of PPE. An essential requirement that must be met by any wearable device is that it must be comfortable for everyday use and must adapt to its user [7].

In some sectors, the conditions in the work environment create risks for the workers, as a result it is necessary to design adapted solutions, according to the risks encountered in that environment [8]. Some notable developments within this field of study are, for example, intelligent protective suits for firefighters, rescue personnel or chemists. We find projects such as PROeTEX [9] or studies such as [10] for fall detection using wearable technology and reducing response time.

\section{PROPOSED MethoD}

Within this framework, several intelligent PPE have been developed, they incorporate wearable electronics and provide advanced functionalities which adapt to conventional products. A series of JSA (Job Safety Analysis) procedures have been performed to identify and group activities/sectors that involve highest accident risk.

Subsequently, we considered the use of different sensors, actuators and protection elements, to appropriately address the identified dangers. The analyzed devices included temperature, impact, piezoelectric, gas, microphone, humidity, pressure, and accelerometer sensors etc. As well as vibration, led and loudspeaker actuators. For communications we have studied bluetooth technology, Wi-Fi, radio frequency, ZigBee, etc. Where applicable, conductive elements, such as conductive fabric, conductive wire, conductive cord, electroluminescent cable, etc., have also been integrated in the garments. Furthermore, we have analyzed the use of lithium batteries, battery charging modules and inductive charging modules. 
For the integration of the devices within the system, different software and hardware options have been studied. Moreover, we have analyzed different sensor networks developed for personal protective equipment (PPE) to meet the expectations of potential users. Likewise, the structural needs and capacities that the system must meet have been examined. To design a comprehensive system, we have also studied the remaining aspects; the required capacities of the security layer, the persistence model, the resource needs, data integration and fusion techniques, etc.

Hence, the designed electronic board has been deployed in a conventional helmet as shown Fig. 3 The board is capable of capturing the following parameters: brightness, impact, temperature, humidity and gases. Furthermore, the device incorporates light actuators in the form of a LED strip on the sides of the helmet, a flashlight that activates automatically if the worker is in poorly lighted areas and a loudspeaker for the detection of audible alarms.

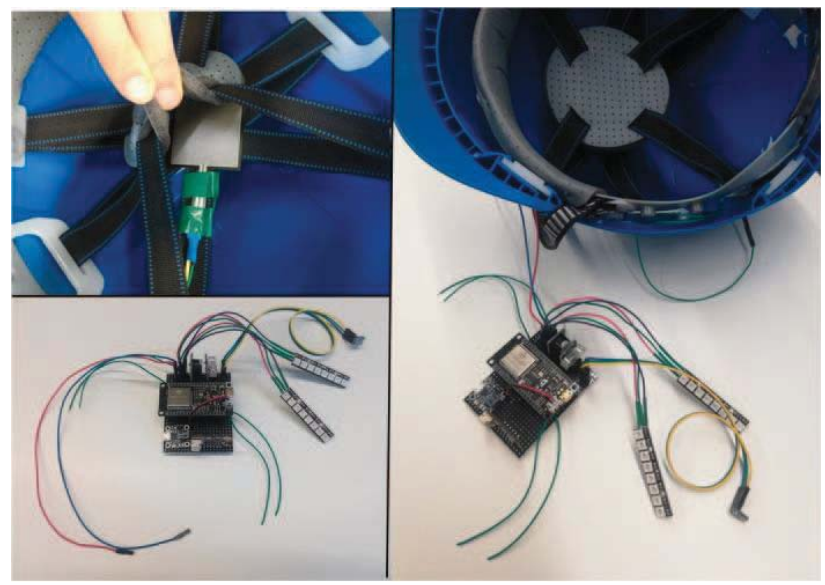

Figure 3. Electronic development and incorporation into the helmet

Finally, a board has been adapted to a conventional belt, it carries an RFID identifier which is used to control the workers access in the building. The belt is also capable of detecting falls because the board integrates accelerometer, this is an ideal place to deploy it because it is where the less sudden movements are captured, thus, an ideal place for detecting falls. The alarms cause the buzzer and vibrator to activate in the bracelet, warning the worker of a danger. Its design makes it comfortable to wear and allows for free movement. As a differentiating feature, it uses conductive elements such as fabric or thread for the interconnection of the devices and the power supply.

In addition, a bracelet is designed to notify the operator of the electronic system about environmental conditions. Likewise, the bracelet gathers information of its environment through the following components: module placed for visualizing the information coming from the helmet and the belt. Furthermore, to notifying the operator is used a vibration module and a speaker. The bracelet consists of a thermocouple temperature sensor, detecting the body temperature of the subject, a photoresistor responsible for suppressing notifications and a heart rate sensor, responsible for measuring the heartbeat. if the information obtained affects the integrity of the individual, notify him about the danger in his environment or body condition in order to take appropriate precautionary measures.

In order to improve the detection of anomalies obtained from the components of the electronic systems, 6 artificial intelligence algorithms are used, which were trained taking an average of 50,000 data collected from each of the electronic systems, which were submitted to Data Cleansing techniques in order to reduce the presence of errors during training.

\section{RESUlTS}

The designed PPE rendered very good results in the performed experimental tests. The tests have demonstrated that the designed elements effectively counteract risks, increasing the safety of workers in the working environment.

We have tested the performance of the sensors, including the accelerometer placed on the belt for which detects falls. We have designed an algorithm based on previous work done by Sorvala [20] which uses the values obtained by the accelerometer to determine if a worker has fallen or not. Fig. 4 shows the simulation of a fall detecting three periods marked with the red boxes. The three periods define the different states; before the fall, during the fall and after the fall. The acceleration values during the fall are significantly higher than the values obtained normally, this is visible on the X-AXIS and Y-AXIS and the period after the fall shows very little acceleration in all axes.

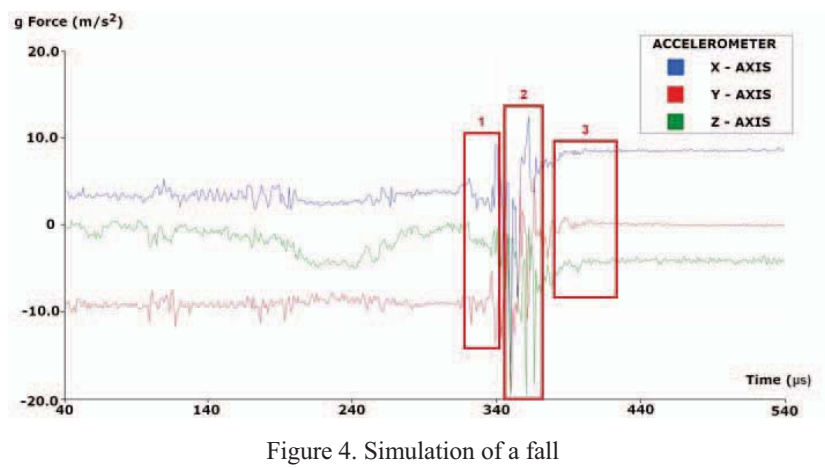

Furthermore, the BME 680 is used to measure gas concentration, air quality, pressure, temperature and humidity It is located on the helmet because it is the nearest place to the respiratory tract. In Fig. 5 we can see the measurements of this sensor.

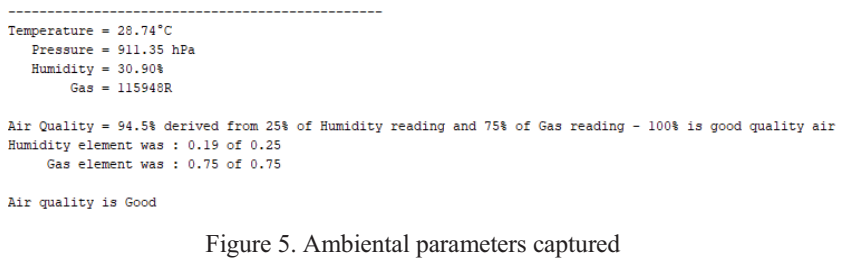

Finally, the graph in Fig. 6 shows the light intensity values taken by a sensor located in the helmet. If the level of light drops below an established threshold represented with a red 
line, the flashlight will automatically be switched on to increase the visibility for the operator.

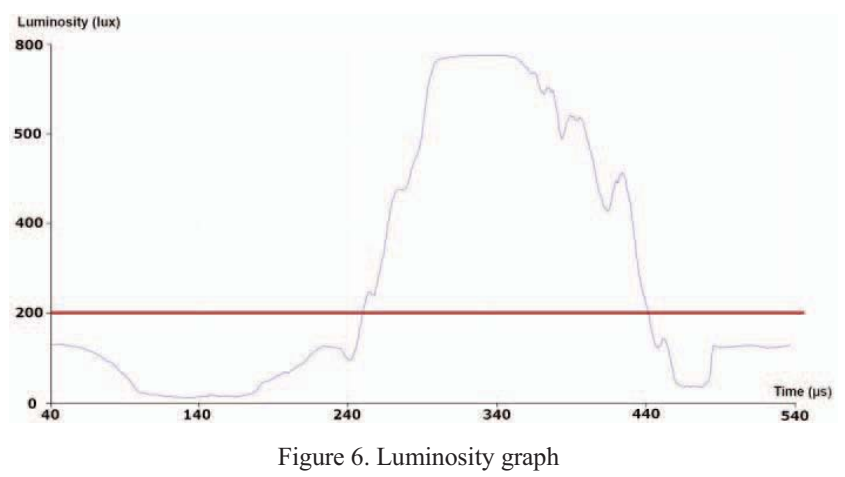

The alarm system implemented on a dedicated server, as shown in Fig. 7, is responsible for notifying the supervisor or manager of the installation about the abnormal conditions in the workers environment, notifying in real time the conditions of luminosity, gas, ambiental pressure, temperature, presence of hits in the worker's helmet, heart rate, body temperature and presence of falls of the worker.

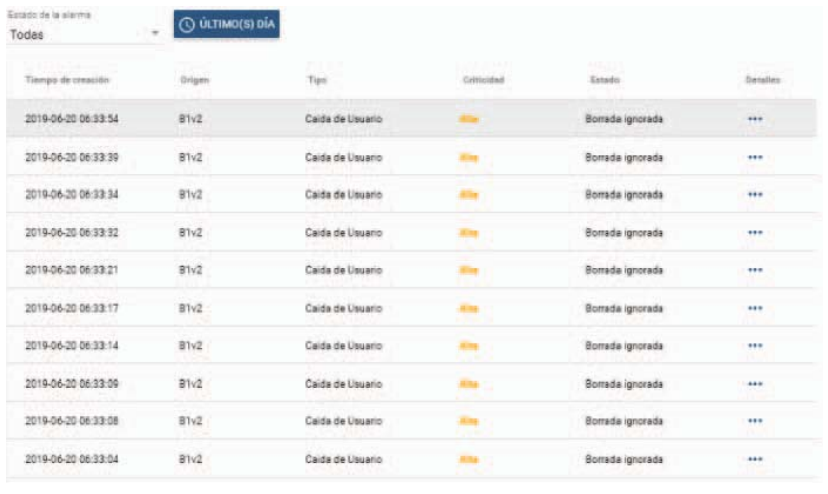

Figure 7. Alarm system notification

In this study, we obtained samples of data from a real environment, when there was a significant variation in the data; a label was added to feed the ML models, the models were programmed in Jupyter with the objective to comparison of artificial intelligence algorithms in the helmet.

In the training data and test phase of the artificial intelligence models, the reliability intervals of the supervised learning algorithms are obtained respectively, as shown in Table 1, highlighting the use of Random Forest (classifier) during the research, with a margin of error of $0.0106 \%$.

TABLE I. COMPARISON OF ARTIFICIAL INTELLIGENCE ALGORITHMS IN THE HELMET

\begin{tabular}{|l|c|}
\hline \multicolumn{1}{|c|}{ Supervised learning model } & Predictive accuracy \\
\hline $\begin{array}{l}\text { Neural Network Artificial } \\
\text { (feedforward) }\end{array}$ & 0.9019 \\
\hline Random Forest (classifier) & 0.989413680781759 \\
\hline Random Forest (regression) & 0.670448109 \\
\hline
\end{tabular}

\begin{tabular}{|l|c|}
\hline \multicolumn{1}{|c|}{ Supervised learning model } & Predictive accuracy \\
\hline Decision Tree & 0.9853420195439739 \\
\hline SVM linear & 0.94 \\
\hline SVM kernel polynomial & 0.49 \\
\hline
\end{tabular}

\section{CONCLUSiOn}

This work has presented a novel system based on the creation of occupational risk prevention devices. Our system combines wireless communication, Smart Textiles and electronic devices. Moreover, the data collected by the system can be visualized by the user on a tablet or a mobile phone. There's currently no other system like this one.

There is therefore a demand for an emergency plan against accidents at work in which the administration, employers and workers coordinate to prevent accidents. It has been proven that the proposed system significantly improves the safety conditions of operators; it is trained with data to ensure early detection and fast response to risk situations. Moreover, new sensors will be investigated as a new means of identification of risks, this will be an important part of the created intelligent fabrics, that is, electronic textiles for physiological and physical monitoring. Thanks to our knowledge of technology in the fields of circuit printing and flexible printed circuit board (PCBs), in a future work we are going to include an improved version of communications model.

In addition, we will create a new control system with new predictive algorithms for risk detection based on Multi-Agent [16][19], data quality [18] and Case based reasoning (CBR) [17]. Moreover, our system provides real-time monitoring, valid for use in different industries.

\section{ACKNOWLEDGMENT}

This work was supported by the Spanish Junta de Castilla y León, Consejería de empleo. Project: UPPER, aUgmented reality and smart personal protective equipment (PPE) for intelligent pRevention of occupational hazards and accessibility INVESTUN/18/SA/0001.

\section{REFERENCES}

[1] Dogan, O., Akcamete, A.: Detecting Falls-from-Height with Wearable Sensors and Reducing Consequences of Occupational Fall Accidents Leveraging IoT. In: Advances in Informatics and Computing in Civil and Construction Engineering. pp. 207-214. Springer International Publishing, Cham (2019).

[2] European Agency for Safety and Health at Work (EUOSHA).Priorities for occupational safety and health research in Europe: 2013-2020. Bilbao: EU-OSHA; 2013.

[3] Podgorski, D., Majchrzycka, K., Dąbrowska, A., Gralewicz, G., I\& Okrasa, M. (2017). Towards a conceptual framework of OSH risk management in smart working environments based on smart PPE, ambient intelligence and the Internet of Things technologies. International Journal of Occupational Safety and Ergonomics, 23(1), 120.

[4] PEROSH. Sustainable workplaces of the future - European research challenges for occupational safety and health. Brussels: PEROSH 
(Partnership for European Research on Occupational Safety and Health); 2012.

[5] Mondal S. Phase change materials for smart textiles - an overview. Appl Therm Eng. 2008;28:1536-1550.

[6] Rocha JG, Goncalves LM, Rocha PF, et al. Energy harvesting from piezoelectric materials fully integrated in footwear. IEEE Trans Ind Electron. 2010;57:813-819.

[7] Tao, X. (Ed.). (2005). Wearable electronics and photonics. Elsevier.

[8] Scott, R. A. (Ed.). (2005). Textiles for protection. Elsevier.

[9] Stephanidis, C. (2012). Human factors in ambient intelligence environments. Handbook of Human Factors and Ergonomics, 13541373.

[10] Schneegass, S., Voit, A.: Gesturesleeve: Using touch sensitive fabrics for gestural input on the forearm for controlling smartwatches. In: Proceedings of the 2016 ACM International Symposium on Wearable Computers. ISWC '16, pp. 108-115. ACM, New York (2016)

[11] Gopalsamy, C., Park, S., Rajamanickam, R., Jayaraman, S.: The wearable motherboard: the first generation of adaptive and responsive textile structures (ARTS) for medical applications. Virtual Real. 4(3), 152-168 (1999)

[12] Amft, O., Habetha, J.: Smart medical textiles for monitoring patients with heart conditions. In: Langenhove, L.v. (ed.) Book chapter in: Smart textiles for medicine and healthcare.Woodhead Publishing Ltd, Cambridge, England, pp. 275-297 (February 2007) ISBN 1845690273

[13] Schneegass, S., Hassib, M., Zhou, B., Cheng, J., Seoane, F., Amft, O., ... \& Schmidt, A. (2015, September). SimpleSkin: towards multipurpose smart garments. In Adjunct Proceedings of the 2015 ACM International Joint Conference on Pervasive and Ubiquitous Computing and
Proceedings of the 2015 ACM International Symposium on Wearable Computers (pp. 241-244). ACM

[14] Choi, S., \& Jiang, Z. (2008). A wearable cardiorespiratory sensor system for analyzing the sleep condition. Expert Systems with Applications, 35(1-2), 317-329. Chen, D., I\& Lawo, M. (2017).

[15] Chen, D., \& Lawo, M. (2017). Smart textiles and smart personnel protective equipment. In Smart Textiles (pp. 333-357). Springer, Cham.

[16] González-Briones, A., Chamoso, P., Yoe, H., I\& Corchado, J. M. (2018). GreenVMAS: virtual organization based platform for heating greenhouses using waste energy from power plants. Sensors, 18(3), 861.

[17] González-Briones, A., Rivas, A., Chamoso, P., Casado-Vara, R., \& Corchado, J. M. (2018, June). Case-based reasoning and agent based job offer recommender system. In The 13th International Conference on Soft Computing Models in Industrial and Environmental Applications (pp. 21-33). Springer, Cham.

[18] Casado-Vara, R., Prieto-Castrillo, F., I\& Corchado, J. M. A game theory approach for cooperative control to improve data quality and false data detection in WSN. International Journal of Robust and Nonlinear Control.

[19] Rodríguez, S., Palomino, C. G., Chamoso, P., Silveira, R. A., I\& Corchado, J. M. (2018, August). How to create an adaptive learning environment by means of virtual organizations. In International Workshop on Learning Technology for Education in Cloud (pp. 199212). Springer, Cham.

[20] Sorvala A., Alasaarela E., Sorvoja H., Myllyla R. A two-threshold fall detection algorithm for reducing false alarms; Proceedings of the 6th International Symposium on Medical Information and Communication Technology (ISMICT); La Jolla, CA, USA. 25-29 March 2012 